\title{
Mitochondrial stress protein HSP60 regulates ER stress-induced hepatic lipogenesis
}

\author{
Ting Xiao', Xiuci Liang1', Hailan Liu1, Feng Zhang1, Wen Meng1 and Fang Hư 1,2,3 \\ 'Department of Metabolism and Endocrinology, The Second Xiangya Hospital, Central South University, Changsha, Hunan, China \\ ${ }^{2}$ National Clinical Research Center for Metabolic Diseases, Changsha, Hunan, China \\ 3Metabolic Syndrome Research Center, Central South University, Changsha, Hunan, China
}

Correspondence should be addressed to F Hu: hu_fang98@csu.edu.cn or to W Meng: 122501006@csu.edu.cn

\begin{abstract}
Endoplasmic reticulum (ER) stress and mitochondrial dysfunction are associated with hepatic steatosis and insulin resistance. Molecular mechanisms underlying ER stress and/ or mitochondrial dysfunction that cause metabolic disorders and hepatic steatosis remain to be fully understood. Here, we found that a high fat diet (HFD) or chemically induced ER stress can stimulate mitochondrial stress protein HSP60 expression, impair mitochondrial respiration, and decrease mitochondrial membrane potential in mouse hepatocytes. HSP60 overexpression promotes ER stress and hepatic lipogenic protein expression and impairs insulin signaling in mouse hepatocytes. Mechanistically, HSP60 regulates ER stress-induced hepatic lipogenesis via the mTORC1-SREBP1 signaling pathway. These results suggest that HSP60 is an important ER and mitochondrial stress cross-talking protein and may control ER stress-induced hepatic lipogenesis and insulin resistance.

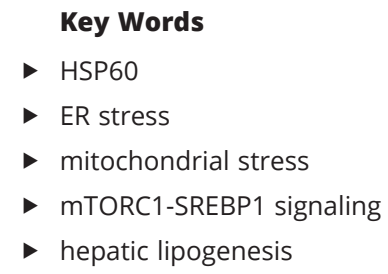

Journal of Molecular Endocrinology (2020) 64, 67-75
\end{abstract}

\section{Introduction}

Endoplasmic reticulum (ER) stress due to high fat diet (HFD) feeding or genetic obesity is associated with hepatic steatosis and insulin resistance (Kammoun et al. 2009, Yoshiuchi et al. 2009, Ye et al. 2010, Meng et al. 2017, Luo et al. 2018). Hepatic steatosis is characterized by an excessive accumulation of lipids that ultimately leads to disruption of tissue architecture and organ dysfunction. However, the precise mechanisms by which ER stress causes metabolic disorders and hepatic steatosis are yet to be fully elucidated. Recent studies have revealed that hepatic mTORC1-SREBP1 (sterol regulatory elementbinding protein 1c) signaling plays a key role in regulating systemic hepatic lipid metabolism (Porstmann et al. 2008, Düvel et al. 2010, Yecies et al. 2011, Owen et al. 2012, Cai et al. 2016). Not only does mTORC1 regulate Srebp-1 gene transcription (Bakan \& Laplante 2012) but it also promotes the transformation of primordial SREBP
(p-SREBP) to functional mature SREBP (m-SREBP) (Han \& Wang 2018). However, the mechanisms underlying ER stress-induced hepatic lipogenesis and steatosis via the mTORC1- SREBP1 signaling pathway are yet to be completely elucidated.

Similar to the stress response in the ER, mitochondrial stress is characterized by a mitochondrial unfolded protein response (UPR ${ }^{\mathrm{mt}}$ ) and initiation of a retrograde stress signaling pathway accompanied by impaired mitochondrial function and membrane potential (Haynes et al. 2007, Broadley \& Hartl 2008, Haynes \& Ron 2010, Hu \& Liu 2011). HSP60, an important mitochondrial stress protein, has been shown to play key roles in the protein synthesis, folding, and delivery of misfolded proteins to proteolytic enzymes in the mitochondrial matrix (Tatsuta 2009, Haynes \& Ron 2010, Hu \& Liu 2011, Marino Gammazza et al. 2018). Recent studies have showed that 
mitochondrial stress is associated with mitochondrial dysfunction and metabolic disorders such as hepatic steatosis, insulin resistance, and type 2 diabetes (Venojarvi et al. 2008, Hu \& Liu 2011, Comert et al. 2019, Einer et al. 2019). ER and mitochondria have partially overlapping functions, and there is strong crosstalk between the two organelles to regulate hepatic steatosis (Haynes \& Ron 2010, Hu \& Liu 2011, Ghemrawi et al. 2018). However, the cause-and-effect relationship between ER and mitochondrial stress in response to nutrients overloading are yet to be clearly elucidated.

In current studies, we showed that HFD induced both ER stress and mitochondrial stress in the liver of C57/B6 mice. In addition, we found that ER stress also induced mitochondrial stress protein HSP60 expression and impaired mitochondrial function and membrane potential in mouse hepatocytes. The overexpression of HSP60 enhanced ER stress and impaired insulin signaling. Importantly, our studies demonstrate HSP60regulated ER stress-induced hepatic lipogenesis via the mTORC1-SREBP1 signaling pathway. Thus, HFD and ER stress-induced HSP60 might be an important ER and mitochondrial stress cross-talking protein that plays a key role in the regulation of hepatic lipid metabolism.

\section{Materials and methods}

\section{Chemicals and antibodies}

ER stress-inducer tunicamycin (TM) and stress blocker tauroursodeoxycholic acid (TUDCA) were purchased from Sigma. The primary antibodies against mouse $\beta$-actin, phospho-Akt (Ser473), AKT, CHOP, phosphoFOXO1(FOXO1-P), FOXO1, mTORC1, p70S6K, and phospho-p70S6K (S6K-P) were obtained from Cell Signaling Technology Inc. The primary antibodies against HSP60, GRP78, SREBP1, fatty acid synthase (FAS), ACC, and phospho-ACC (ACC-P) were obtained from Santa Cruz Biotechnology. The horseradish peroxidase-conjugated secondary antibodies were obtained from Promega.

\section{Cell culture, treatment, and transfection}

HepIR mouse liver cells were cultured in MEM $\alpha$ (Gibco) culture medium supplemented with $4 \%$ fetal bovine serum (FBS; Gibco) and $1 \%$ penicillin/streptomycin (Gibco) in $37^{\circ} \mathrm{C}$ and $5 \% \mathrm{CO}_{2}$ incubator. ER stress was induced by treating cells with tunicamycin (TM) $(0.1 \mathrm{nM})$ for $24 \mathrm{~h}$. For HSP60 overexpression, HepIR cells were infected with control or HSP60 expressing vector by lentivirus for $8 \mathrm{~h}$, and then washed and incubated for another $48 \mathrm{~h}$ before collection. To knock down endogenous Hsp60 gene expression, HepIR cells were transfected with Hsp60-siRNA (forward: 5'-GGAAGUCCCA AAGUAA CAATT-3'; reverse: 5'-UUG UUACUUUGGGACUUCCTT-3') or control NC-siRNA (forward: 5'-UUCUCCGAACGUGUCACGUTT-3'; reverse 5'-ACGUGACACGUUCGGAGATT-3') using Lipofectamine 2000 (Invitrogen). Cells were collected after $48 \mathrm{~h}$ transfection.

\section{Animal studies}

Six-week-old male mice (C57BL/6J) were purchased from Slac Laboratory Animal Inc. (Shanghai, China) and housed in a temperature-controlled environment with a 12:12 h light/dark cycle. The mice had free access to food and water ad libitum. After a one week adaptive period, the mice were randomly divided into two weightmatched groups and fed with either a high fat diet (HFD) (60\% kcal; \#12492) or a control diet (10\% kcal; \#12540B) (Research Diets, Inc., New Brunswick, USA). After 8 weeks of feeding, the animals were killed and their livers were rapidly isolated, followed by immediate freezing in liquid nitrogen and storing at $-80^{\circ} \mathrm{C}$ before analyses. ER stress was induced by i.p. administration of TM (Sigma). Briefly, a single i.p. injection of TM $(1 \mathrm{mg} / \mathrm{kg})$ or equal volumes of vehicle (saline) were applied to the male mice, and $16 \mathrm{~h}$ after injection the mice were killed and the tissues were collected. All protocols for animal use and maintenance were approved by the Central South University Animal Care and Use Committee.

\section{Western blots}

For protein extraction, approximately $30 \mathrm{mg}$ of frozen tissue was homogenized in $400 \mu \mathrm{l}$ RIPA buffer (Beyotime Institute of Biotechnology, Shanghai, China). Extracts were spun down and the fat layer and cell debris were removed. Protein concentration was determined by the BCA kit. Equal amounts of proteins from each sample were loaded and separated by SDS-PAGE. Proteins were transferred to polyvinylidene difluoride membrane and incubated with a blocking buffer (5\% BSA in $20 \mathrm{mM}$ Tris- $\mathrm{HCl}, \mathrm{pH} 7.5,137 \mathrm{mM} \mathrm{NaCl}$, and 0.1\% Tween 20) for $1 \mathrm{~h}$ at room temperature and then incubated with primary antibodies at $4^{\circ} \mathrm{C}$ overnight. The membrane was incubated with secondary antibodies (1:5000 to 1:10,000 dilution) for $1 \mathrm{~h}$ at room temperature and detected with enhanced chemiluminescence (Bio-Rad). 


\section{Mitochondrial membrane potential measurement}

Mitochondrial membrane potential $(\Delta \psi \mathrm{m})$ was measured using the fluorescent probe JC-1 $\left(5,5^{\prime}, 6,6^{\prime}\right.$-tetrachloro1,1',3,3'-tetraethyl-benzimidazol carbocyanine iodide) (Invitrogen) as described previously (Woollacott \& Simpson 2001). JC-1 is a cationic dye that is accumulated in mitochondria following membrane potential. Briefly, after treatment with or without $0.1 \mu \mathrm{g} / \mathrm{mL}$ TM for $24 \mathrm{~h}$, cells were trypsinised and resuspended with Krebs-Ringer HEPES buffer $\left(120 \mathrm{mM} \mathrm{NaCl}, 1.9 \mathrm{mM} \mathrm{CaCl}_{2}, 4.6 \mathrm{mM}\right.$ $\mathrm{KCl}, 25 \mathrm{mM}$ HEPES, $1 \mathrm{mM} \mathrm{MgSO}$, $1.2 \mathrm{mM} \mathrm{KH}_{2} \mathrm{PO}_{4}, 1 \%$ $(w / v)$ BSA, pH 7.4). Then $1 \times 10^{6}$ cells were incubated with $1 \mu \mathrm{g} / \mathrm{ml} \mathrm{JC} 1$ at $37^{\circ} \mathrm{C}$ for $20 \mathrm{~min}$, immediately followed by the measuring of red (excitation $488 \mathrm{~nm}$, emission 575 $\mathrm{nm}$ ) to green fluorescence (excitation $488 \mathrm{~nm}$, emission $530 \mathrm{~nm}$ ) by a flow cytometry system (BD Biosciences, USA). Mitochondrial depolarization was achieved by treating cells with carbonyl cyanide 4-(trifluoromethoxy) phenylhydrazone (FCCP; $10 \mu \mathrm{M})\left(20 \mathrm{~min}, 37^{\circ} \mathrm{C}\right)$, an uncoupling agent that abolishes the $\Delta \psi \mathrm{m}$.

\section{Oxygen consumption}

Mitochondrial oxygen consumption in intact cells were measured with the Seahorse Bioscience XF-24 analyzer (Seahorse Bioscience, Billerica, MA, USA) and reported as oxygen consumption rate (OCR) as described previously (Brand \& Nicholls 2011). HepIR cells were seeded at 15000 cells/well into XF-24 culture microplates and cultured overnight at $37^{\circ} \mathrm{C}$ with $5 \% \mathrm{CO}_{2}$. During the $24 \mathrm{~h}$ post-treatment with or without TM, the medium was replaced with pre-warmed $600 \mu \mathrm{l}$ of sodium carbonate-free DMEM for $1 \mathrm{~h}$. Each experimental condition was analyzed using four to six biological replicates. The following reagents including $1 \mu \mathrm{M}$ oligomycin (Sigma-Aldrich), $1 \mu \mathrm{M}$ FCCP (Sigma-Aldrich) or $1 \mu \mathrm{M}$ rotenone (SigmaAldrich) were added to block state III respiration, induce uncoupling, or shut down mitochondrial respiration, respectively. Data were normalized to protein content.

\section{Oil red 0 staining}

Cultured cells were fixed with $4 \%$ paraformaldehyde for 30 min and washed with PBS. The fixed cells were stained with $1 \%$ Oil red $\mathrm{O}$ for $1 \mathrm{~h}$ at room temperature and rinsed with PBS thrice. Oil red O-stained lipid-laden cells in different experimental groups were observed with an Olympus microscope.

\section{Statistical analysis}

All data were reported as average \pm s.E.M. Differences between control and treatment were analyzed by unpaired, two-tailed Student's $t$-test, and statistical significance was set as $P<0.05$.

\section{Results}

ER stress-induced mitochondrial stress protein HSP60 expression in liver of mice

Recent studies have revealed that ER stress and mitochondrial dysfunction were induced in the liver of HFD-fed mice accompanied by hepatic lipogenesis and steatosis (Hu \& Liu 2011, Yecies et al. 2011, Ghemrawi et al. 2018, Luo et al. 2018). While ER and mitochondria are subjected to distinct regulations in response to stress, these two organelles are connected at multiple levels during the stress response (Hu \& Liu 2011). As the mechanism of HFD and ER stress-induced-hepatic lipogenesis is still poorly understood, we sought to determine whether mitochondrial stress contributes to lipid accumulation in the liver of mice. As expected, HFD induced the high expression of GRP78 and C/EBP-homologous protein (CHOP), which are ER stress signaling marker proteins (Fig. 1A). Interestingly, we found that HSP60, an important marker of mitochondrial stress, was also greatly increased in the liver of HFD-fed mice (Fig. 1A), suggesting that HFD induces ER stress as well as mitochondrial stress in the liver of mice.

In order to determine whether ER stress induced mitochondrial stress in vivo, we injected TM, an inhibitor of the ER-specific calcium ATPase, to induce ER stress in C57/B6 mice. As expected, the expression levels of GRP78 and CHOP were significantly increased. Meanwhile, the expression of mitochondrialstress protein HSP60in theliver of TM-injected mice also increased significantly (Fig. 1B). Thus, the results show that both HFD and ER stress induced HSP60 expression in the liver of C57/B6 mice.

\section{ER stress impaired mitochondrial function and membrane potential in mouse hepatocytes}

To further determine whether ER stress directly induces mitochondrial dysfunction in a cellular autonomous manner, we used TM to induce ER stress and examined the expression levels of HSP60 in HepIR cells. As expected, the use of TM led to a significant increase in the level of ER stress in hepatocytes, which was demonstrated by increased CHOP and GRP78 expression levels (Fig. 2A).

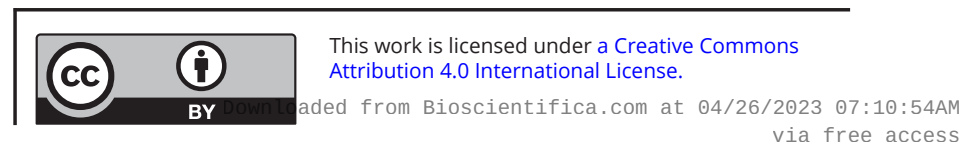


A
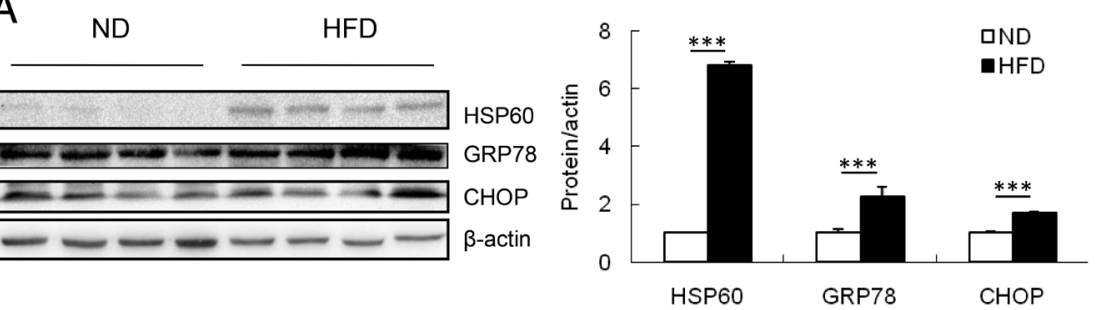

B
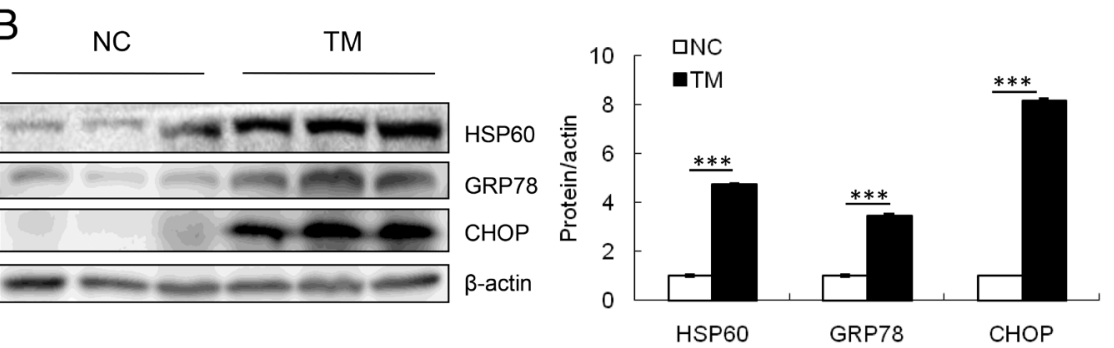

\section{Figure 1}

ER stress-induced mitochondrial stress protein HSP60 expression in liver of mice. (A) Western blot analyses of the protein levels of HSP60, GRP78, and CHOP in liver of mice after a 8-week HFD or ND feeding regimen starting at 8 weeks of age. (B) The protein levels of HSP60, GRP78, and CHOP in liver of mice after i.p. injection of $1 \mathrm{mg} / \mathrm{kg}$ TM for 16 h. Data are presented as mean \pm S.E.M. $\star \star \star P<0.001$. HFD: high fat diet; ND: normal diet; TM: tunicamycin.
Concurrent with the increased level of ER stress, the expression level of HSP60 also increased in the TM-treated cells compared with that in the control HepIR cells, while the use of ER stress-reducing chemical chaperone TUDCA caused a decline in TM-induced ER stress protein (CHOP and GRP78) and mitochondrial stress protein HSP60 expression (Fig. 2A). Therefore, the results show that ER stress directly induced mitochondrial stress in mouse hepatocytes.

To determine whether ER stress alters mitochondrial respiration, we measured the oxygen consumption in HepIR cells that were treated either with or without TM. Through TM treatment, the basal and FCCP-stimulated maximal respiration was significantly suppressed in HepIR cells (Fig. 2B). JC-1 is a cationic fluorescent dye that is accumulated in mitochondria in a potentialdependent manner. A gradual increase in JC-1 green fluorescence and loss of orange fluorescence represents cells with compromised mitochondrial membrane potential. We then went on to measure the effect of ER stress on mitochondrial membrane potential in HepIR cells and found that the JC-1 green fluorescence increased significantly in HepIR cells treated with TM, and the percentage of such cells increased from $2.97 \%$ to $35.8 \%$ (Fig. 2C). The mitochondrial transmembrane potential (indicated by FL-2/FL-1 ratio) was decreased in early
A

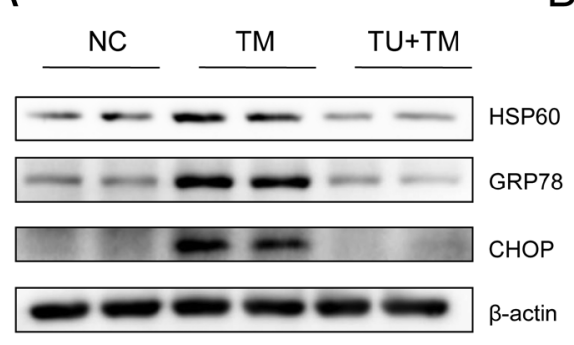

C

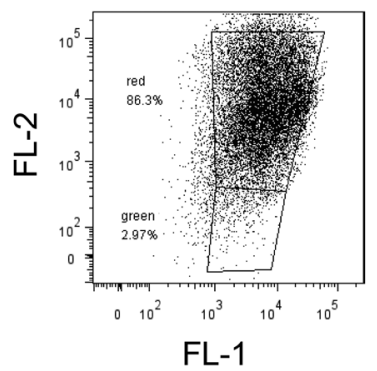

NC
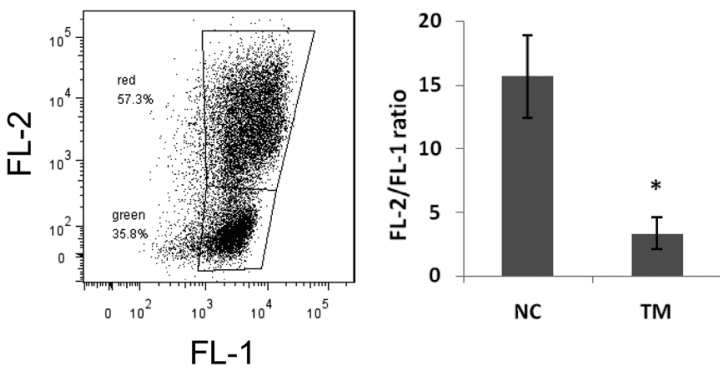

Figure 2

ER stress impaired mitochondrial function and membrane potential in mouse hepatocytes. (A) Western blot analyses of the protein levels of HSP60, GRP78, and CHOP in HepIR cells treated with or without TM $(0.1 \mathrm{nM})$ for $24 \mathrm{~h}$, followed by treatment with or without TUDCA $(100 \mu \mathrm{M})$ for 1 h. (B) OCR of HepIR cells treated by TM. $\mathrm{VO}_{2}$ consumption was normalized to protein content. (C) Flow cytometry analysis for mitochondrial membrane potential in HepIR cells treated with or without TM. Data are presented as mean \pm S.E.M. $\star P<0.05 ; * * P<0.01$. OCR: oxygen consumption rate; OL: oligomycin; Rot: rotenone; Anti: antimycin; TM: tunicamycin; TUDCA: tauroursodeoxycholic acid. https://jme.bioscientifica.com https://doi.org/10.1530/JME-19-0207 (c) 2020 The authors Published by Bioscientifica Ltd. Printed in Great Britain

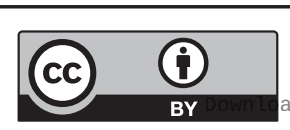

This work is licensed under a Creative Commons Attribution 4.0 International License. 
apoptotic cells by the ER stress inducer TM (Fig. 2C). Thus, these results suggest that ER stress impaired mitochondrial respiration and membrane potential.

\section{HSP60 overexpression induced ER stress and impaired insulin signaling in mouse hepatocytes}

While numerous studies have demonstrated that a close biochemical and physiological connection exists between ER and mitochondria (Franzini-Armstrong 2007, Giorgi et al. 2009, Beller et al. 2010), an interesting question that remains unanswered is the cause-and-effect relationship between ER and mitochondrial stress. Since our studies showed that ER stress induced mitochondrial stress, we then went on to determine whether mitochondrial stress can induce ER stress. Overexpression of HSP60 stimulated ER stress levels in the hepatocytes of mice greatly, as demonstrated by increased CHOP and GRP78 expression levels (Fig. 3A). It was reported that insulin sensitivity was regulated by cross-talking between mitochondria and ER during stress (Hu \& Liu 2011, Ghemrawi et al. 2018). To decide whether overexpression of HSP60 regulates insulin sensitivity, we treated HSP60-overexpressed hepatocytes with or without insulin (Fig. 3B). We found that insulin stimulated the phosphorylation of AKT and FOXO1, and this effect was impaired greatly by overexpressing HSP60 in HepIR cells (Fig. 3B), indicating that HSP60 overexpression impaired insulin signaling and insulin sensitivity in mouse hepatocytes.

\section{ER stress induced hepatic lipogenesis via HSP60- mediated mTORC1-SREBP1 signaling pathway}

As depicted in Fig. 1A and B, HFD and TM induced both ER and mitochondrial stress in the liver of mice. To determine whether ER stress-stimulated mitochondrial stress affects hepatic lipid metabolism, we measured the level of SREBP1 protein, a key transcription factor in promoting hepatic lipogenesis. We found that SREBP1 levels were increased in the liver of HFD-fed mice (Fig. 4A) and TM-treated mice (Fig. 4B). Interestingly, in agreement with these findings, overexpression of HSP60 stimulated the hepatic SREBP1, FAS experssion (Fig. 4C), as well as the mTORC1 signaling in mouse hepatocytes. Furthermore, Oil red O staining experiments showed that overexpression of HSP60 promoted hepatic lipogenesis (Fig. 4D).

To further define the role of HSP60 in the regulation of hepatic lipid metabolism, we knocked out HSP60 under the action of TM in mouse hepatocytes. We found that treating mouse hepatocytes with TM led to an increase in the expression of HSP60, and a marked increase in mTOR signaling and SREBP1 associated lipogenesis, as demonstrated by enhanced S6K-P and FAS, and increased m-SREBP1 expression, while the knockdown of HSP60 suppressed these protein levels, indicating inactivation of the mTORC1-SREBP1 signaling pathway (Fig. 4E). On the other hand, the knockdown of HSP60 blocked ER stress-induced hepatic lipogenesis as determined by Oil red $\mathrm{O}$ staining (Fig. 4F). Together, these results suggest that hepatic HSP60 could be a critical effector to the downstream of ER stress for the induction of mTORC1SREBP1 signaling and maybe involved in mTORC1SREBP1-regulated hepatic lipid metabolism.

\section{Discussion}

In this study, we have confirmed that ER stress is important in the regulation of hepatic lipogenesis, which was consistent with the previous reports about ER stressinduced hepatic steatosis and insulin resistance (Hu \& Liu 2011, Yecies et al. 2011, Chen et al. 2017, Ghemrawi et al. 2018, Han \& Wang 2018, Luo et al. 2018). More importantly, we have also shown that ER stress induced mitochondrial stress protein HSP60 expression and impaired mitochondrial function in mouse hepatocytes

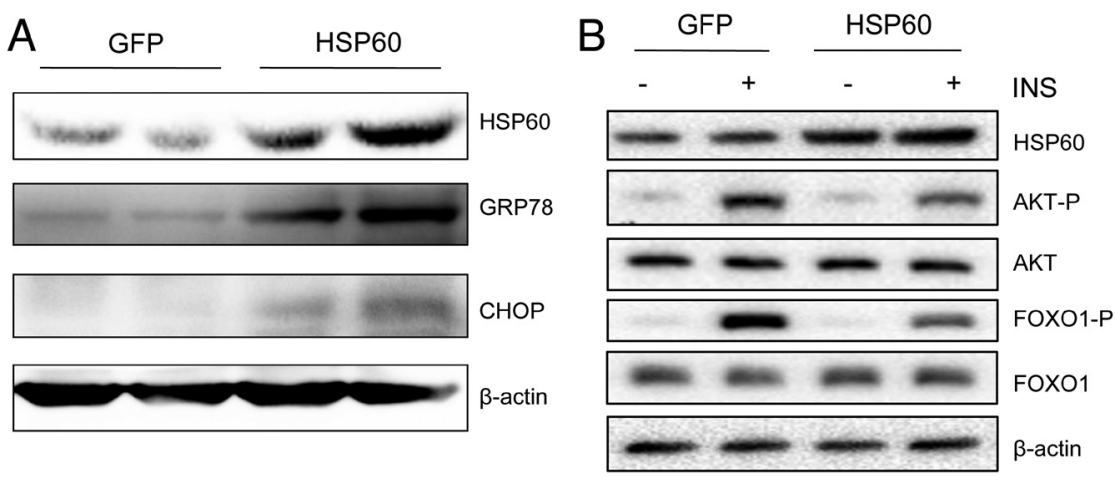

Figure 3

HSP60 overexpression induced ER stress and impaired insulin signaling in mouse hepatocytes. (A) Western blot analyses of the protein levels of HSP60, GRP78, and CHOP in mouse hepatocytes overexpressing HSP60. (B) Mouse hepatocytes were infected with lentivirus encoding HSP60, followed by treatment with or without insulin (10 $\mathrm{nM}$ ) for $10 \mathrm{~min}$, and expressions of the indicated proteins were analyzed by Western blot. https://jme.bioscientifica.com https://doi.org/10.1530/JME-19-0207 (c) 2020 The authors Published by Bioscientifica Ltd. Printed in Great Britain

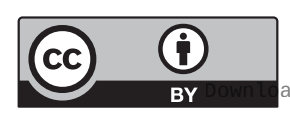

This work is licensed under a Creative Commons Attribution 4.0 International License. ded from Bioscientifica.com at 04/26/2023 07:10:54AM 
A

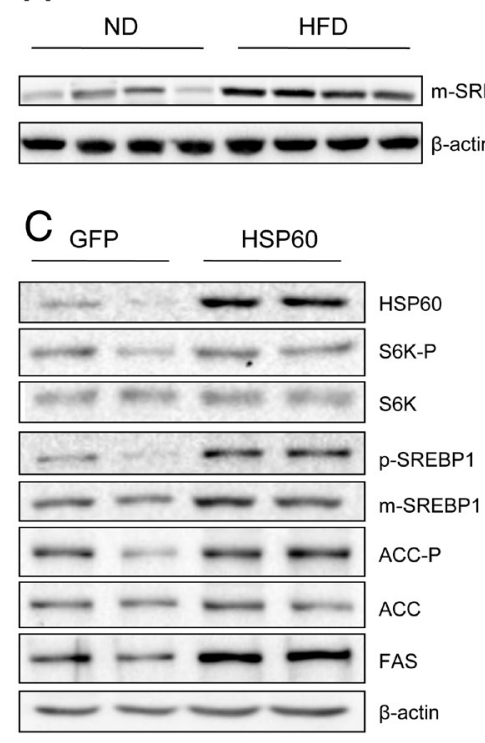

B

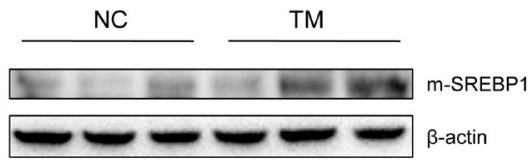

D
HSP60

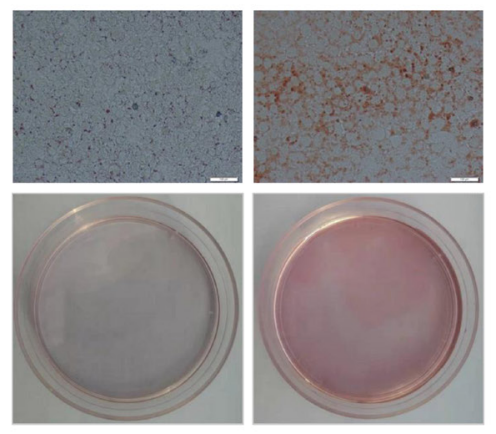

$\frac{\text { E NC }}{--++++--\frac{T M}{++}}$

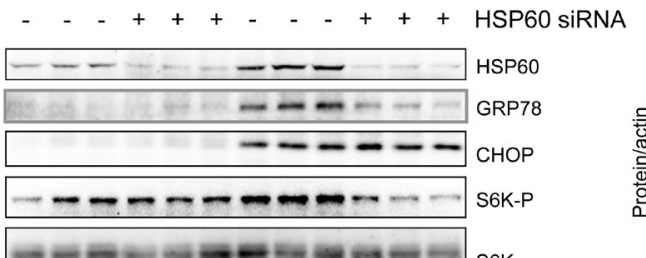
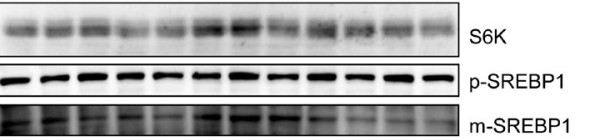

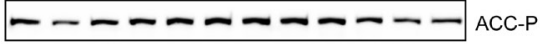
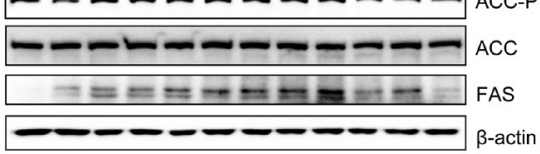

$\mathrm{F}$

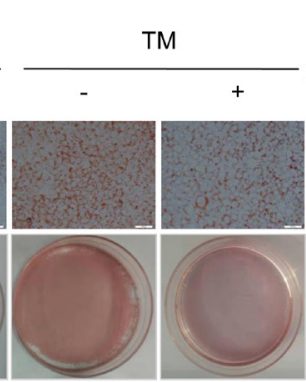

口NC-ncsiRNA

口NC-HSP60siRNA

口TM-nCSIRNA

-TM-HSP60siRNA
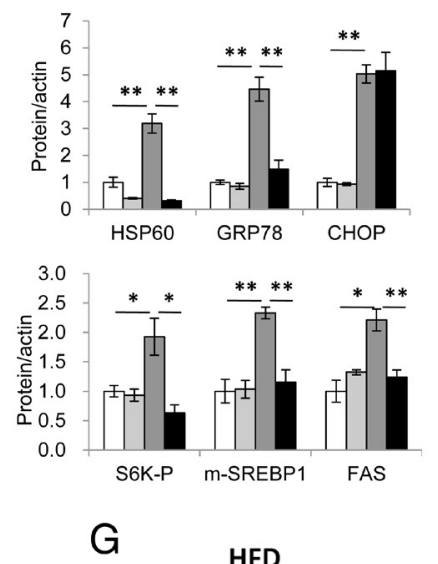

G Hsp60

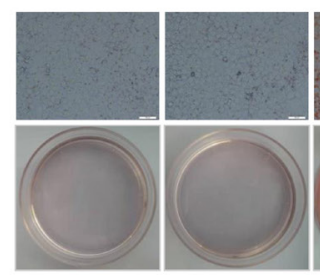
siRNA

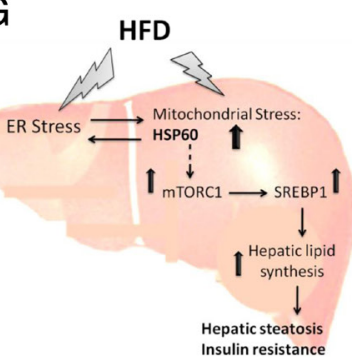

\section{Figure 4}

ER stress induced hepatic lipogenesis via HSP60-mediated mTORC1-SREBP1 signaling pathway. (A) Western blot analyses of the protein level of SREBP1 in liver of mice after a 8-week HFD or ND feeding regimen starting at 8 weeks of age. (B) The protein level of SREBP1 in liver of mice after i.p. injection of 1 $\mathrm{mg} / \mathrm{kg}$ TM for $16 \mathrm{~h}$. (C) Western blot analyses of the phosphorylation and protein levels of S6K, ACC, FAS, and primordial SREBP (p-SREBP) and mature SREBP ( $m$-SREBP) in mouse hepatocytes overexpressing HSP60. (D) Oil red O staining in mouse hepatocytes overexpressing HSP60 (Scale bar = 100 m). (E) Mouse hepatocytes were treated with Hsp60 siRNA or their control siRNA, followed by treatment with or without TM ( $0.1 \mathrm{nM}$ ) for $24 \mathrm{~h}$, and expressions of the indicated proteins were analyzed by Western blot. (F) Mouse hepatocytes were treated with Hsp60 siRNA or their control siRNA, followed by treatment with or without TM $(0.1 \mathrm{nM})$ for $24 \mathrm{~h}$ and stained with Oil red O (Scale bar $=100 \mu \mathrm{m})$. (G) A proposed model showing the role of HSP60 in HFD and ER stress-induced hepatic steatosis and insulin resistance. The HFD and ER stress-induced mitochondrial stress protein HSP60 promotes lipid accumulation via mTORC1-SREBP1 signaling pathway, leading to hepatic steatosis and insulin resistance. The dashed arrow indicates direct or indirect action. A full colour version of this figure is available at https://doi.org/10.1530/JME-19-0207.

https://jme.bioscientifica.com

https://doi.org/10.1530/JME-19-0207 (c) 2020 The authors Published by Bioscientifica Ltd. Printed in Great Britain

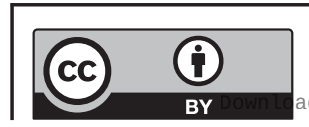

This work is licensed under a Creative Commons Attribution 4.0 International License. 
(Fig. 2A and C). HSP60 overexpression deteriorated ER stress and impaired insulin signaling (Fig. 3A and B). Furthermore, we have demonstrated that the knockdown of HSP60 inhibited ER stress-induced mTORC1-SREBP1 signaling pathway is essential in regulating hepatic lipid metabolism (Fig. 4E and F). We first identified ER stressinduced mitochondrial stress protein as the potential mediator of mTORC1-SREBP1 signaling in hepatic lipid metabolism. Our results revealed a previously unrecognized mechanism, in which HFD and ER stress induced hepatic steatosis and insulin resistance.

Previous studies have shown that mitochondrial stress caused by obesity and/or metabolic dysfunction induces the upregulation of mitochondrial stress protein and accumulation of ROS to disrupt membrane potential and uncoupling of OXPHOS, leading to decreased mitochondrial respiration and mitochondrial dysfunction (Biswas et al. 1999, Amuthan et al. 2002, Haynes \& Ron 2010, Hu \& Liu 2011). As a chaperone protein, HSP60 plays a wide range of functions in the mitochondrion, including the folding of newly synthesized proteins, the appropriate translocation and folding of proteins within organelles, and the refolding of aggregating or misfolded proteins (Hartl \& Hayer-Hartl 2002, Tatsuta 2009, Hu \& Liu 2011). New evidence has emphasized the link between ER and mitochondria during stress response (Zhao et al. 2002, Haynes \& Ron 2010, Hu \& Liu 2011). Mitochondrial stress is induced by calcium and ROS/anti-oxidative signaling in response to ER stress (Hu \& Liu 2011, Ghemrawi et al. 2018). Consistently, our study showed that ER stress directly induced mitochondrial stress protein HSP60 expression (Fig. 2A) and impaired mitochondrial function and membrane potential in the hepatocytes of mice (Fig. 2B and C). On the other hand, overexpression of HSP60 also induced ER stress by increasing GRP78 and CHOP protein levels (Fig. 3A). These results suggested a further functional connection between ER and mitochondria in response to stress.

Interestingly, the knockdown of HSP60 inhibited GRP78 protein expression greatly but had no effect on CHOP expression in the hepatocytes of mice (Fig. 4E). Unfolded or misfolded proteins are recognized by GRP78, which is accompanied by the initiation of ER stress, which ultimately leads to increased CHOP expression and apoptosis (Ghemrawi et al. 2018). It is possible that HSP60 may be involved in the initiation regulation of ER stress, but not in the ER stress-induced apoptosis signaling pathway. Therefore, the underlying mechanism requires further investigation.

A number of recent reports have shown that mitochondrial dysfunction plays an important role in insulin resistance (Perez-Carreras et al. 2003, Pessayre \& Fromenty 2005, Mitchell et al. 2009). Consistent with these results, we found that increased mitochondrial stress protein HSP60 inhibited the insulin signaling pathway and impaired insulin sensitivity (Fig. 3B), demonstrating that HSP60 may have a negative effect on hepatic insulin sensitivity. Accumulation of HSP60 can induce insulin resistance in skeletal muscle cells by stimulating the release of pro-inflammatory cytokines (Marker et al. 2012, Habich \& Sell 2015). In addition, high HSP60 serum concentrations were also reported in type 2 diabetic subjects (Dasu et al. 2010). These results suggest that stressinduced HSP60 might stimulate inflammation resulting in metabolic dysfunction and insulin resistance, but the underlying mechanism still requires further investigation. Interestingly, it was reported that rosiglitazone enhanced insulin sensitivity and mitochondrial HSP60 level, which resulted in improved glucose tolerance in the adipocytes of ob/ob mice (Wilson-Fritch et al. 2004), suggesting that mitochondrial HSP60, in the UPR ${ }^{\mathrm{mt}}$ pathway, may have distinct functions in different tissues in vivo.

HFD or ER stress has been well-documented to induce SREBP1 activation associated with hepatic lipogenesis and steatosis (Yoshiuchi et al. 2009, Yecies et al. 2011, Luo et al . 2018). Consistent with these findings, we also found that HFD or ER stress can induce SREBP1 signaling activation, accompanied by increased hepatic lipogenesis (Fig. 4A, B, $\mathrm{E}$ and $\mathrm{F}$ ). A recent study showed that mitochondrial stress is linked to lipid homeostasis in C. elegans and human cells (Kim et al. 2016). Mitochondrial dysfunction is associated with hepatic lipogenesis and steatosis (Perez-Carreras et al. 2003, Pessayre \& Fromenty 2005, Mitchell et al. 2009). Consistent with HFD or ER stress-induced SREBP1 signaling activation, HSP60 overexpression activated mTORC1-SREBP1 signaling and hepatic lipogenesis (Fig. 4C and D), whereas the knockdown of HSP60 suppressed ER stress-induced mTORC1-SREBP1 signaling and hepatic lipogenesis (Fig. 4E and F), suggesting that HSP60 might be involved in ER stress-induced mTORC1SREBP1 signaling associated with lipogenesis and steatosis (Fig. 4G). In the future, a liver-specific, genetically modified animal model might be necessary to clarify the role of HSP60 in lipid metabolism in vivo. In addition, whether HSP60 directly induces mTORC1-SREBP1 signaling needs further investigation.

To summarize, we have discovered that ER stress can induce mitochondrial stress, and the mitochondrial stress protein HSP60 is a novel regulator of mTORC1-SREBP1 signaling in control of hepatic lipid metabolism. Our findings indicate that targeting HSP60 might provide a

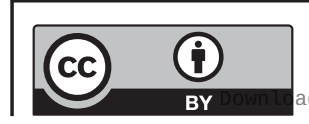

This work is licensed under a Creative Commons Attribution 4.0 International License. ded from Bioscientifica.com at 04/26/2023 07:10:54AM 
new strategy to counteract HFD- and ER stress-induced hepatic steatosis and insulin resistance.

\section{Declaration of interest}

The authors declare that there is no conflict of interest that could be perceived as prejudicing the impartiality of the research reported.

\section{Funding}

This work was supported by grants from the National Nature Science Foundation of China $(91957113,31871180$, and 31471131 to $\mathrm{F} \mathrm{H}$; and 81800758 to W M).

\section{Author contribution statement}

$\mathrm{T} X$ and $\mathrm{W} M$ performed collection, analysis, and assembly of data and prepared the first draft of the manuscript; $X \mathrm{~L}, \mathrm{H} \mathrm{L}$, and $\mathrm{F} Z$ performed data collection; F H and W M performed conceptualization and design, data analysis and interpretation, manuscript writing, financial support, and approved the final manuscript. All authors reviewed and approved the manuscript. $\mathrm{FH}$ is the guarantor of this work and, as such, had full access to all the data in the study and takes responsibility for the integrity and accuracy of the data.

\section{Acknowledgement}

We thank Shirley Pan for language editing.

\section{References}

Amuthan G, Biswas G, Ananadatheerthavarada HK, Vijayasarathy C, Shephard HM \& avadhani NG 2002 Mitochondrial stress-induced calcium signaling, phenotypic changes and invasive behavior in human lung carcinoma A549 cells. Oncogene 21 7839-7849. (https:// doi.org/10.1038/sj.onc.1205983)

Bakan I \& Laplante M 2012 Connecting mTORC1 signaling to SREBP-1 activation. Current Opinion in Lipidology 23 226-234. (https://doi. org/10.1097/MOL.0b013e328352dd03)

Beller M, Thiel K, Thul PJ \& Jackle H 2010 Lipid droplets: a dynamic organelle moves into focus. FEBS Letters $\mathbf{5 8 4} 2176-2182$. (https://doi. org/10.1016/j.febslet.2010.03.022)

Biswas G, Adebanjo OA, Freedman BD, Anandatheerthavarada HK, Vijayasarathy C, Zaidi M, Kotlikoff M \& Avadhani NG 1999 Retrograde $\mathrm{Ca} 2+$ signaling in $\mathrm{C} 2 \mathrm{C} 12$ skeletal myocytes in response to mitochondrial genetic and metabolic stress: a novel mode of inter-organelle crosstalk. EMBO Journal 18 522-533. (https://doi. org/10.1093/emboj/18.3.522)

Brand MD \& Nicholls DG 2011 Assessing mitochondrial dysfunction in cells. Biochemical Journal 435 297-312. (https://doi.org/10.1042/ BJ20110162)

Broadley SA \& Hartl FU 2008 Mitochondrial stress signaling: a pathway unfolds. Trends in Cell Biology 18 1-4. (https://doi.org/10.1016/j. tcb.2007.11.003)

Cai H, Dong LQ \& Liu F 2016 Recent advances in adipose mTOR signaling and function: therapeutic prospects. Trends in Pharmacological Sciences 37 303-317. (https://doi.org/10.1016/j.tips.2015.11.011)

Chen H, Bai J, Dong F, Fang H, Zhang Y, Meng W, Liu B, Luo Y, Liu M, Bai Y, et al. 2017 Hepatic DsbA-L protects mice from diet-induced hepatosteatosis and insulin resistance. FASEB Journal 31 2314-2326. (https://doi.org/10.1096/fj.201600985R) Printed in Great Britain
Comert C, Fernandez-Guerra P \& Bross P 2019 A cell model for HSP60 deficiencies: modeling different levels of chaperonopathies leading to oxidative stress and mitochondrial dysfunction. Methods in Molecular Biology 1873 225-239. (https://doi.org/10.1007/978-1-4939-8820-4_14)

Dasu MR, Devaraj S, Park S \& Jialal I 2010 Increased toll-like receptor (TLR) activation and TLR ligands in recently diagnosed type 2 diabetic subjects. Diabetes Care 33 861-868. (https://doi.org/10.2337/ dc09-1799)

Düvel K, Yecies JL, Menon S, Raman P, Lipovsky AI, Souza AL, Triantafellow E, Ma Q, Gorski R, Cleaver S, et al. 2010 Activation of a metabolic gene regulatory network downstream of mTOR complex 1. Molecular Cell 39 171-183. (https://doi.org/10.1016/j. molcel.2010.06.022)

Einer C, Leitzinger C, Lichtmannegger J, Eberhagen C, Rieder T, Borchard S, Wimmer R, Denk G, Popper B, Neff F, et al. 2019 A highcalorie diet aggravates mitochondrial dysfunction and triggers severe liver damage in Wilson disease rats. Cellular and Molecular Gastroenterology and Hepatology 7 571-596. (https://doi.org/10.1016/ j.jcmgh.2018.12.005)

Franzini-Armstrong C 2007 ER-mitochondria communication. How privileged? Physiology 22 261-268. (https://doi.org/10.1152/ physiol.00017.2007)

Ghemrawi R, Battaglia-Hsu SF \& Arnold C 2018 Endoplasmic reticulum stress in metabolic disorders. Cells 7 E63. (https://doi.org/10.3390/ cells7060063)

Giorgi C, De Stefani D, Bononi A, Rizzuto R \& Pinton P 2009 Structural and functional link between the mitochondrial network and the endoplasmic reticulum. International Journal of Biochemistry and Cell Biology 41 1817-1827. (https://doi.org/10.1016/j.biocel.2009.04.010)

Habich C \& Sell H 2015 Heat shock proteins in obesity: links to cardiovascular disease. Hormone Molecular Biology and Clinical Investigation 21 117-124. (https://doi.org/10.1515/hmbci-2014-0040)

Han J \& Wang Y 2018 mTORC1 signaling in hepatic lipid metabolism. Protein and Cell 9 145-151. (https://doi.org/10.1007/s13238-0170409-3)

Hartl FU \& Hayer-Hartl M 2002 Molecular chaperones in the cytosol: from nascent chain to folded protein. Science 295 1852-1858. (https://doi.org/10.1126/science.1068408)

Haynes CM \& Ron D 2010 The mitochondrial UPR - protecting organelle protein homeostasis. Journal of Cell Science 123 3849-3855. (https://doi.org/10.1242/jcs.075119)

Haynes CM, Petrova K, Benedetti C, Yang Y \& Ron D 2007 ClpP mediates activation of a mitochondrial unfolded protein response in C. elegans. Developmental Cell 13 467-480. (https://doi.org/10.1016/j. devcel.2007.07.016)

Hu F \& Liu F 2011 Mitochondrial stress: a bridge between mitochondrial dysfunction and metabolic diseases? Cellular Signalling $\mathbf{2 3}$ 1528-1533. (https://doi.org/10.1016/j.cellsig.2011.05.008)

Kammoun HL, Chabanon H, Hainault I, Luquet S, Magnan C, Koike T, Ferre P \& Foufelle F 2009 GRP78 expression inhibits insulin and ER stress-induced SREBP-1c activation and reduces hepatic steatosis in mice. Journal of Clinical Investigation 119 1201-1215. (https://doi. org/10.1172/JCI37007)

Kim HE, Grant AR, Simic MS, Kohnz RA, Nomura DK, Durieux J, Riera CE, Sanchez M, Kapernick E, Wolff S, et al. 2016 Lipid biosynthesis coordinates a mitochondrial-to-cytosolic stress response. Cell 166 1539.e16-1552.e16. (https://doi.org/10.1016/j. cell.2016.08.027)

Luo L, Jiang W, Liu H, Bu J, Tang P, Du C, Xu Z, Luo H, Liu B, Xiao B, et al. 2018 De-silencing Grb10 contributes to acute ER stress-induced steatosis in mouse liver. Journal of Molecular Endocrinology $\mathbf{6 0}$ 285-297. (https://doi.org/10.1530/JME-18-0018)

Marino Gammazza A, Macaluso F, Di Felice V, Cappello F \& Barone R 2018 Hsp60 in skeletal muscle fiber biogenesis and homeostasis: from physical exercise to skeletal muscle pathology. Cells 7 Hsp60. (https://doi.org/10.3390/cells7120224)

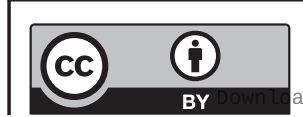

This work is licensed under a Creative Commons Attribution 4.0 International License. ded from Bioscientifica.com at 04/26/2023 07:10:54AM 
Marker T, Sell H, Zillessen P, Glode A, Kriebel J, Ouwens DM, Pattyn P, Ruige J, Famulla S, Roden M, et al. 2012 Heat shock protein 60 as a mediator of adipose tissue inflammation and insulin resistance. Diabetes 61 615-625. (https://doi.org/10.2337/db10-1574)

Meng W, Liang X, Chen H, Luo H, Bai J, Li G, Zhang Q, Xiao T, He S, Zhang Y, et al. 2017 Rheb inhibits beiging of white adipose tissue via PDE4D5-dependent downregulation of the cAMP-PKA signaling pathway. Diabetes 66 1198-1213. (https://doi.org/10.2337/db16-0886)

Mitchell C, Robin MA, Mahrouf-Yorgov M, Mayeuf A, Mansouri A, Fromenty B \& Gilgenkrantz H 2009 Protection against hepatocyte mitochondrial dysfunction delays fibrosis progression in mice. Hepatology 50 823a-823a. (https://doi.org/10.2353/ ajpath.2009.090332)

Owen JL, Zhang Y, Bae SH, Farooqi MS, Liang G, Hammer RE, Goldstein JL \& Brown MS 2012 Insulin stimulation of SREBP-1c processing in transgenic rat hepatocytes requires p70 S6-kinase. PNAS 109 16184-16189. (https://doi.org/10.1073/pnas.1213343109)

Perez-Carreras M, Del Hoyo P, Martin MA, Rubio JC, Martin A, Castellano G, Colina F, Arenas J \& Solis-Herruzo JA 2003 Defective hepatic mitochondrial respiratory chain in patients with nonalcoholic steatohepatitis. Hepatology 38 999-1007. (https://doi. org/10.1053/jhep.2003.50398)

Pessayre D \& Fromenty B 2005 Nash: a mitochondrial disease. Journal of Hepatology 42 928-940. (https://doi.org/10.1016/j.jhep.2005.03.004)

Porstmann T, Santos CR, Griffiths B, Cully M, Wu M, Leevers S, Griffiths JR, Chung YL \& Schulze A 2008 SREBP activity is regulated by mTORC1 and contributes to Akt-dependent cell growth. Cell Metabolism 8 224-236. (https://doi.org/10.1016/j.cmet.2008.07.007)

Tatsuta T 2009 Protein quality control in mitochondria. Journal of Biochemistry 146 455-461. (https://doi.org/10.1093/jb/mvp122)

Venojarvi M, Aunola S, Puhke R, Marniemi J, Hamalainen H, Halonen JP, Lindstrom J, Rastas M, Hallsten K, Nuutila P, et al. 2008
Exercise training with dietary counselling increases mitochondrial chaperone expression in middle-aged subjects with impaired glucose tolerance. BMC Endocrine Disorders 8 3. (https://doi. org/10.1186/1472-6823-8-3)

Wilson-Fritch L, Nicoloro S, Chouinard M, Lazar MA, Chui PC, Leszyk J, Straubhaar J, Czech MP \& Corvera S 2004 Mitochondrial remodeling in adipose tissue associated with obesity and treatment with rosiglitazone. Journal of Clinical Investigation 114 1281-1289. (https:// doi.org/10.1172/JCI21752)

Woollacott AJ \& Simpson PB 2001 High throughput fluorescence assays for the measurement of mitochondrial activity in intact human neuroblastoma cells. Journal of Biomolecular Screening 6 413-420. (https://doi.org/10.1177/108705710100600607)

Ye R, Jung DY, Jun JY, Li J, Luo S, Ko HJ, Kim JK \& Lee AS 2010 Grp78 heterozygosity promotes adaptive unfolded protein response and attenuates diet-induced obesity and insulin resistance. Diabetes $\mathbf{5 9}$ 6-16. (https://doi.org/10.2337/db09-0755)

Yecies JL, Zhang HH, Menon S, Liu S, Yecies D, Lipovsky AI, Gorgun C, Kwiatkowski DJ, Hotamisligil GS, Lee CH, et al. 2011 Akt stimulates hepatic SREBP1c and lipogenesis through parallel mTORC1dependent and independent pathways. Cell Metabolism 14 21-32. (https://doi.org/10.1016/j.cmet.2011.06.002)

Yoshiuchi K, Kaneto H, Matsuoka TA, Kasami R, Kohno K, Iwawaki T, Nakatani Y, Yamasaki Y, Shimomura I \& Matsuhisa M 2009 Pioglitazone reduces ER stress in the liver: direct monitoring of in vivo ER stress using ER stress-activated indicator transgenic mice. Endocrine Journal 56 1103-1111. (https://doi.org/10.1507/endocrj. k09e-140)

Zhao Q, Wang J, Levichkin IV, Stasinopoulos S, Ryan MT \& Hoogenraad NJ 2002 A mitochondrial specific stress response in mammalian cells. EMBO Journal 21 4411-4419. (https://doi. org/10.1093/emboj/cdf445)

Received in final form 21 November 2019

Accepted 4 December 2019

Accepted Manuscript published online 4 December 2019 (c) 2020 The authors Published by Bioscientifica Ltd. Printed in Great Britain

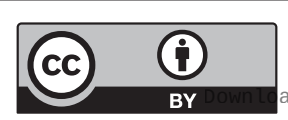

This work is licensed under a Creative Commons Attribution 4.0 International License.

ded from Bioscientifica.com at 04/26/2023 07:10:54AM 